\title{
Competition between anisotropy and superconductivity in organic and cuprate superconductors
}

\author{
T. Schneider \\ Physik-Institut der Universität Zürich, Winterthurerstrasse 190, \\ CH-8057 Zürich, Switzerland
}

\begin{abstract}
We analyze the empirical correlation between the zero temperature penetration depth $\lambda_{c}(0)$ and the corresponding normal state DC conductivity $\sigma_{c}^{D C}$, measured slightly above the transition temperature $T_{c}$, in different classes of quasi two-dimensional superconductors, including cuprates and organics. For this purpose we invoke the scaling theory of quantum and finite temperature critical phenomena. Important implications are: Superconductivity in the organic and cuprate superconductors is a genuine three dimensional (3D) phenomenon. The competition between anisotropy and superconductivity destroys the latter in the $2 \mathrm{D}$ limit even in the ground state. The data uncovers the flow to quantum criticality, including the 2D quantum superconductor to insulator (2D-QSI) and the 3D quantum superconductor to normal state (3D-QSN) transition. This flow gives a clear perspective of the regimes where quantum fluctuations are essential and mean-field treatments fail. Thus, a detailed account of the flow from mean-field to 2D-QSI criticality in organics and the crossover from the 2D-QSI to the 3D-QSN critical point in cuprates is a challenge for microscopic theories attempting to solve the puzzle of superconductivity in these materials.
\end{abstract}

The formation of the superconducting condensate in elemental metals and their alloys is well understood within the BCS-Eliashberg mean-field theory of superconductivity. Here pairing and phase coherence (superfluidity) set in at the same temperature. Applicability to highly anisotropic quasi-two-dimensional superconductors, including organic and cuprate superconductors is challenged by the evidence for strong thermal and quantum fluctuations in the normal and superconducting state of these layered materials 11 . 4 . In this paper we concentrate on interplane properties, emerging from the empirical correlation between the zero temperature penetration depth $\lambda_{c}(0)$, due to supercurrents flowing perpendicular to the layers, and the corresponding DC conductivity $\sigma_{c}^{D C}\left(T_{c}^{+}\right)$, measured slightly above the transition temperature $T_{c}$ [5. (6). To provide an understanding of this empirical correlation and to explore the implications, we invoke the scaling theory of finite temperature and quantum critical phenomena. An essential role plays the anisotropy. It is shown that the experimental data points, in the $\left(\lambda_{c}(0), \sigma_{c}^{D C}\left(T_{c}^{+}\right)\right)$-plane, including organic, cuprate and conventional superconductors, map the flow to or away from limiting critical behavior. We identify the two dimensional quantum superconductor to insulator (2D-QSI), the three dimensional quantum superconductor to normal state (3D-QSN), the finite temperature 3D-XY transition and its mean-field counterpart as the essential limiting critical points. Since the flow to 2D-QSI criticality implies increasing anisotropy, our analysis reveals that superconductivity in the organics and cuprates is a genuine $3 \mathrm{D}$ phenomenon and that the competition between anisotropy and superconductivity destroys the latter in the 2D limit even in the ground state. Moreover the flow to 2D-QSI and 3D-QSN criticality and the associated crossovers give a clear perspective of the regimes where quantum fluctuations are essential and mean-field treatments fail. Thus, a detailed account of the flow from mean-field to 2D-QSI criticality in organics and the crossover from the 2D-QSI to the 3D-QSN critical point is a challenge for microscopic theories attempting to solve the puzzle of superconductivity in these materials. For a detailed discussion of the generic phase diagram of cuprate superconductors and the emerging critical points we refer to recent reviews [1.,8].

In Fig.11 we displayed $\lambda_{c}(0)$ versus $\sigma_{c}^{D C}\left(T_{c}^{+}\right)$for a variety of cuprate, organic and conventional superconductors, collected by Dordevic et al. [5, 6] and Motohashi et al. [8]. With the exception of overdoped cuprates, we observe that the magnitude of $\lambda_{c}(0)$ is systematically suppressed with the increase of the normal state conductivity $\sigma_{c}^{D C}$. This feature appears to be consistent with the relation

$$
\lambda_{c}(0)=\Omega_{s}\left(\sigma_{c}^{D C}\left(T_{c}^{+}\right)\right)^{-1 / 2}, \Omega_{s}=\left(\frac{c^{2} \tau_{t r}\left(T_{c}^{+}\right)}{4 \pi}\right)^{1 / 2},
$$

resulting from the standard expressions for the normal state conductivity $\sigma_{c}^{D C}(T)=n e^{2} \tau_{t r}(T) / m_{c}$ and the definition of the London penetration depth $1 / \lambda_{c}^{2}(T=0)=\left(4 \pi n e^{2}\right) /\left(m_{c} c^{2}\right) \cdot \tau_{t r}$ is the mean scattering time of the electrons in transport properties with number density $\mathrm{n}$ and effective mass $m_{c}$. This relationship also agrees with the meanfield predictions for bulk superconductors in the dirty limit [46] and layered BCS superconductors, treated as weakly coupled Josephson junction $47-49$, where $\Omega_{s}=\left(\hbar c^{2} /\left(4 \pi^{2} \Delta(0)\right)\right)^{1 / 2}$ and $\Delta(0)$ denotes the zero temperature energy gap. Thus, $\lambda_{c}(0) \propto\left(\sigma_{c}^{D C}\left(T_{c}^{+}\right)\right)^{-1 / 2}$ is a characteristic feature of conventional superconductors, where fluctuations do 
not play an essential role. Indeed, the dotted line in Fig.1, which is the mean-field scaling form (1), captures the trend emerging from the data of conventional superconductors, as well as of $\mathrm{MgB}_{2}$ and $\mathrm{Sr}_{2} \mathrm{RuO}_{4}$, remarkably well. Prominent and systematic deviations occur for the highly anisotropic organic and underdoped cuprate superconductors. As $\lambda_{c}(0)$ increases with the reduction of the normal state conductivity $\sigma_{c}^{D C}$, we observe that the data tends to fall onto two branches, consistent with

$$
\lambda_{c}(0)=\Omega_{s}\left(\sigma_{c}^{D C}\right)^{-x}, x \approx 3 / 4
$$

The most prominent deviations from these trends occur for overdoped cuprates. In particular the data for overdoped $\mathrm{YBa}_{2} \mathrm{Cu}_{3} \mathrm{O}_{7-\delta}(\mathrm{YBCO})$ exhibit an upturn, suggesting a crossover to a power law of the form (2) but with a negative exponent $x$. Note that this crossover appears to be correlated with the disappearance of the pseudogap in the overdoped regime.

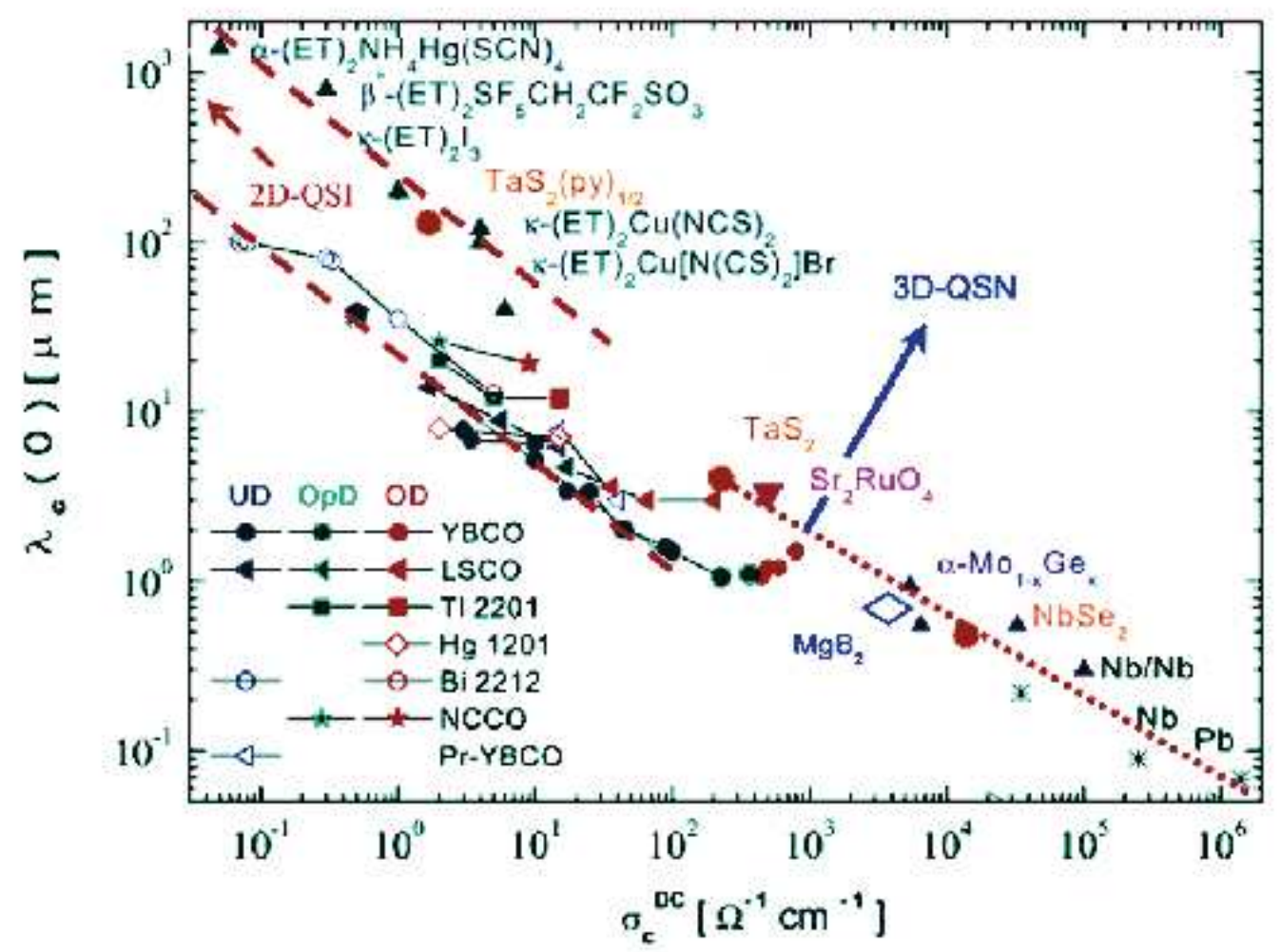

FIG. 1. $\lambda_{c}(0)$ versus $\sigma_{c}^{D C}$ for a variety of superconductors. $\mathrm{YBa}_{2} \mathrm{Cu}_{3} \mathrm{O}_{7-\delta}(\mathrm{YBCO})$ [9] 13$], \mathrm{La}_{2-x} \mathrm{Sr}_{x} \mathrm{CuO} \mathrm{O}_{4+\delta}(\mathrm{LSCO})$ [11, 14, 15], $\mathrm{HgBa}_{2} \mathrm{CuO}_{4+\delta}(\mathrm{Hg} 1201)$ [16], $\mathrm{Tl}_{2} \mathrm{Ba}_{2} \mathrm{CuO}_{6+\delta}$ (Tl2201) [17 [19], $\mathrm{Bi}_{2} \mathrm{Sr}_{2} \mathrm{CaCu}_{2} \mathrm{O}_{8+\delta}\left(\mathrm{Bi}_{22} 212\right)$ [8, 20], $\mathrm{Nd}_{2-x} \mathrm{Ce}_{x} \mathrm{CuO}{ }_{4+\delta}$ (NCCO) 21]. Blue points - underdoped (UD), green -optimally doped (OpD) and red -overdoped (OD). Transition metal dichalcogenides 222 27]; (ET) 2 X compounds 28 36]; $\mathrm{Sr}_{2} \mathrm{RuO}_{4}$ [37,38]; $\mathrm{MgB}_{2}$ 39 41]; $\mathrm{Nb}$ [42, 43]; $\mathrm{Pb}$ [43]; $\mathrm{Nb}$ Josephson junctions [4]; $\alpha-\mathrm{Mo}_{1-x} \mathrm{Ge}_{x}$ [45]. The dotted line is Eq.(1) and the dashed ones Eq.(2). The red arrows indicate the flow to 2D-QSI and for overdoped $\mathrm{YBa}_{2} \mathrm{Cu}_{3} \mathrm{O}_{7-\delta}$ to 3D-QSN criticality, respectively.

An essential ingredient for an understanding of the empirical correlations between $\lambda_{c}(0)$ and $\sigma_{c}^{D C}$ is the anisotropy. For cuprates it is well established that doping and substitution affect the anisotropy $\gamma$ dramatically [1] 0 ]. For cuprates it is well established that doping and substitution affect the anisotropy $\gamma$ dramatically [1].7]. In tetragonal cuprates it is defined as the ratio $\gamma=\xi_{a b} / \xi_{c}$ of the correlation lengths parallel $\left(\xi_{a b}\right)$ and perpendicular $\left(\xi_{c}\right)$ to CuO $\mathrm{O}_{2}$ layers (abplanes). In the superconducting state it can also be expressed as the ratio $\gamma=\lambda_{c} / \lambda_{a b}$ of the London penetration depths due to supercurrents flowing perpendicular $\left(\lambda_{c}\right)$ and parallel $\left(\lambda_{a b}\right)$ to the ab-planes. Approaching a nonsuperconductor to superconductor transition $\xi$ diverges, while in a superconductor to nonsuperconductor transition $\lambda$ tends to infinity. In both cases, however, $\gamma$ remains finite as long as the system exhibits anisotropic but genuine 3D behavior. There 
are two limiting cases: $\gamma=1$ characterizes isotropic 3D- and $\gamma=\infty$ 2D-critical behavior. An instructive model where $\gamma$ can be varied continuously is the anisotropic 2D Ising model [50. When the coupling in the $\mathrm{y}$ direction goes to zero, $\gamma=\xi_{x} / \xi_{y}$ becomes infinite, the model reduces to the $1 \mathrm{D}$ case and $T_{c}$ vanishes. In the GinzburgLandau description of layered superconductors the anisotropy is related to the interlayer coupling. The weaker this coupling is, the larger $\gamma$ is. The limit $\gamma=\infty$ is attained when the bulk superconductor corresponds to a stack of independent slabs of thickness $d_{s}$. With respect to experimental work, a considerable amount of data is available on the chemical composition dependence of $\gamma$. At $T_{c}$ it can be inferred from resistivity $\left(\gamma=\xi_{a b} / \xi_{c}=\sqrt{\rho_{c} / \rho_{a b}}\right)$ and magnetic torque measurements, while in the superconducting state it follows from magnetic torque and penetration depth $\left(\gamma=\lambda_{c} / \lambda_{a b}\right)$ data. Experimentally it is known that as the dopant concentration is reduced, $\gamma_{T_{c}}$ and $\gamma_{T=0}$ increase systematically, and tend to diverge in the underdoped limit. Here $T_{c}$ vanishes and the cuprates undergo a 2D-QSI transition, dominated by quantum fluctuations. For this reason the scaling theory of critical phenomena should apply. At finite temperatures and in the normal state it predicts [1, [7]

$$
\sigma_{a b}^{D C} \propto \frac{\xi_{\tau}}{\xi_{c}}, \sigma_{c}^{D C} \propto \frac{\xi_{c} \xi_{\tau}}{\xi_{a b}^{2}}
$$

where $\xi_{\tau}$ is the correlation length associated with the critical dynamics. At $T_{c}$ the ratio is then simply given by the anisotropy

$$
\frac{\sigma_{c}^{D C}}{\sigma_{a b}^{D C}}=\left(\frac{\xi_{c}}{\xi_{a b}}\right)^{2}=\left(\frac{1}{\gamma_{T_{c}}}\right)^{2}
$$

On the other hand, at zero temperature the penetration depths and anisotropy are related by

$$
\lambda_{c}(0)=\gamma_{T=0} \lambda_{a b}(0)
$$

Close to 2D-QSI criticality, the scaling theory of quantum critical phenomena applies 1, $1,51$. . Here $T_{c}, \gamma_{T}$ and $\lambda_{a b}(0)$ scale as [1,7, 52

$$
T_{c} \propto\left(\lambda_{a b}(0)\right)^{-2} \propto \delta^{\bar{\nu} z}, \quad \gamma_{T_{c}}=\gamma_{T_{c}, 0} \delta^{-\bar{\nu}}, \gamma_{T=0}=\gamma_{0,0} \delta^{-\bar{\nu}}, \lambda_{a b}(0)=\lambda_{a b, 0}(0) \delta^{-\overline{z \nu} / 2}
$$

where $\bar{\nu}$ is the correlation length, $z$ the dynamic critical exponent of the 2D-QSI transition and $\delta$ measures the distance from this critical point. $\gamma_{T_{c}, 0}, \gamma_{0,0}$ and $\lambda_{a b, 0}(0)$ denote the critical amplitudes close to 2D-QSI criticality. Another essential property of this critical point is that for any finite $T_{c}$ the in-plane areal conductivity is always larger than [1, 51 .

$$
\sigma_{a b}^{D C} d_{s} \approx \frac{4 e^{2}}{h}
$$

where $d_{s}$ is the thickness of the independent sheets. Thus close to 2D-QSI criticality the normal state D.C. conductivity $\sigma_{c}^{D C}$, evaluated above but close to $T_{c}$, and zero temperature penetration depth $\lambda_{c}(0)$ scale then as

$$
\lambda_{c}(0)=\Omega_{s} \sigma_{c}\left(T_{c}^{+}\right)^{-(2+z) / 4}, \quad \Omega_{s} \approx \gamma_{0,0} \lambda_{a b, 0}(0)\left(\frac{4 e^{2}}{\gamma_{T_{c}, 0} d_{s} h}\right)^{(2+z) / 4}
$$

In cuprates there is mounting evidence for a 2D-QSI transition with $z=1$ [1, 52, 53 . This value is also consistent with the theoretical prediction for a disordered bosonic system with long-range Coulomb interaction. Here the loss of superfluidity is due to the localization of the pairs, which is ultimately responsible for the transition [54,55. The resulting exponent $(2+z) / 2=3 / 4$ coincides with that in Eq.(2), yielding the dashed lines in Fig.11. Since organic superconductors undergo superconductor to insulator transitions [56], are highly anisotropic and $\gamma$ can be varied over a rather extended interval, i.e. $\gamma_{T_{c}}=180$ for $\kappa$ - $(\mathrm{ET})_{2} \mathrm{Cu}\left[\mathrm{N}(\mathrm{CS})_{2}\right] \mathrm{Br}, \gamma_{T_{c}}=350$ for $\kappa$ - $(\mathrm{ET})_{2} \mathrm{Cu}(\mathrm{NCS})_{2}$ [57] and $\gamma_{T_{c}}=210^{3}$ for $\alpha$ - (BEDT-TTF $)_{2} \mathrm{NH}_{4} \mathrm{Hg}(\mathrm{SCN})_{4}$ [58, it becomes clear that the organics line in Fig.1 marks the flow to 2D-QSI criticality, where the scaling relation (8) applies, as well. This interpretation is also confirmed by the empirical linear relationship between $T_{c}$ and $1 / \lambda_{a b}^{2}(0)$ for various cuprate and organic superconductors [59,60], consistent with the scaling relation (6). It is a characteristic property of nearly two dimensional systems [51,1]. An instructive example is the onset of superfluidity in ${ }^{4} \mathrm{He}$ films adsorbed on disordered substrates, where the linear relationship between $T_{c}$ and areal superfluid density is well confirmed 61]. Moreover, the existence of a threshold in-plane conductivity $\sigma_{a b}^{D C} d_{s}$ (Eq.(ఫ) is also well documented for $\mathrm{Zn}$-substituted $\mathrm{La}_{2-x} \mathrm{Sr}_{x} \mathrm{CuO}_{4} \mathrm{and}_{\mathrm{YBa}} \mathrm{Cu}_{3} \mathrm{O}_{7-\delta}$ 
[62] and underdoped $\mathrm{YBa}_{2} \mathrm{Cu}_{3} \mathrm{O}_{7-\delta}$ [63]. What remains to be shown is wether or not Eq. (8) yields for $\Omega_{s}$ the correct order of magnitude. Considering $\mathrm{La}_{2-x} \mathrm{Sr}_{x} \mathrm{CuO}_{4}$, where $\gamma_{0,0} \approx 1.63, \gamma_{T_{c}, 0} \approx 2, d_{s} \approx 6.6 A$ and $\lambda_{a b, 0}(0) \approx 740 A$ [7] we obtain for $z=1, \Omega_{s} \approx 24 \mu \mathrm{m}(\Omega \mathrm{cm})^{3 / 4}$, which is reasonably close to $\Omega_{s} \approx 18 \mu \mathrm{m}(\Omega \mathrm{cm})^{3 / 4}$, the value of the cuprates line in Fig.11, which matches the data points of underdoped $\mathrm{La}_{2-x} \mathrm{Sr}_{x} \mathrm{CuO}_{4}$. Unlike layered BCS superconductors where $\Omega_{s}$ is determined by the gap and therefore by $T_{c}$ (Eq.(??)), this scale is mainly fixed by the critical amplitude $\lambda_{a b, 0}(0)$ of the zero temperature in-plane penetration depth and the effective thickness of the layers $d_{s}$ at 2D-QSI criticality. For this reason $\Omega_{s}$ does not adopt a universal value. Thus taken together, the interpretation that the dashed lines in Fig.1 mark the flow of underdoped cuprate and organic superconductors to 2D-QSI criticality is well established. From the relationship

$$
T_{c} \propto\left(\frac{1}{\lambda_{a b}(0)}\right)^{2} \propto\left(\frac{1}{\lambda_{c}(0)}\right)^{2 z /(2+z)} \propto\left(\sigma_{c}^{D C}\left(T_{c}^{+}\right)\right)^{z / 2} \propto \gamma_{T_{c}}^{-z} \propto \gamma_{T=0}^{-z},
$$

derived from Eqs.(1) to (8), it then follows that $T_{c}$ and $\sigma_{c}^{D C}\left(T_{c}^{+}\right)$vanish, while $\lambda_{c}(0), \lambda_{a b}(0)$ and the anisotropy $\gamma$ diverge at 2D-QSI criticality. It is clear from this scaling relation how superconductivity competes with anisotropy and the associated enhancement of fluctuations. It implies that in organic and underdoped cuprate superconductors, a no vanishing transition temperature and superfluid density in the ground state is unalterably linked to a finite anisotropy. This raises serious doubts that $2 \mathrm{D}$ models are potential candidates to explain superconductivity in these materials. Moreover, since $\sigma_{a b}^{D C}$ is always larger than $4 e^{2} / h d_{s}$ (Eq.(7)), the observed generic upturn of $\rho_{c}(T)$ in underdoped cuprates [62]63], is according to Eq. (44), yielding $\rho_{c}\left(T_{c}^{+}\right)=\gamma_{T_{c}}^{2} / \sigma_{a b}^{D C}\left(T_{c}^{+}\right) \approx \gamma_{T_{c}}^{2} h / 4 e^{2}$, a 3D-2D crossover phenomenon and with that a characteristic signature of the flow to 2D-QSI criticality.

Next we turn to the behavior of overdoped cuprates. Experimentally it is known that in the overdoped limit $T_{c}$ tends to zero, the anisotropy $\gamma$ remains finite, while $\lambda_{a b}(0)$ and with that $\lambda_{c}(0)$ diverges. This follows from the $\mu$ SR data for $\mathrm{Y}_{0.8} \mathrm{Ca}_{0.2} \mathrm{Ba}_{2}\left(\mathrm{Cu}_{1-y} \mathrm{Zn}_{y}\right) \mathrm{O}_{7-\delta}\left(\mathrm{Y}_{0.8} \mathrm{Ca}_{0.2}-123\right), \mathrm{Tl}_{0.5-y} \mathrm{~Pb}_{0.5+y} \mathrm{Sr}_{2} \mathrm{Ca}_{1-x} \mathrm{Y}_{x} \mathrm{Cu}_{2} \mathrm{O}_{7}(\mathrm{Tl}-1212)$ [64] and $\mathrm{TlBa}_{2} \mathrm{CuO}_{6+\delta}(\mathrm{Tl}-2201)$ [65], where the heavily overdoped regime was attained. Since $\gamma$ remains finite a 3D quantum superconductor to normal state (QSN) transition occurs. $\xi_{\tau}$ scales as $\xi_{\tau} \propto \xi_{a b}^{z_{c l}}$, where $z_{c l}$ is the critical exponent of the finite temperature dynamics. In this case the scaling theory predicts [1],8]

$$
\sigma_{c}^{D C} \propto \frac{\xi_{c} \xi_{\tau}}{\xi_{a b}^{2}} \propto \frac{\xi_{\tau}}{\xi_{a b} \gamma_{T_{c}}} \propto \xi_{a b}^{z_{c l}-1} \propto \delta^{-\bar{\nu}\left(z_{c l}-1\right)}, \quad \lambda_{c}(0) \propto \delta^{-\frac{\bar{\nu}}{2}(1+z)}
$$

so that

$$
\lambda_{c}(0) \propto\left(\sigma_{c}\left(T_{c}^{+}\right)\right)^{\frac{1+z}{2\left(z_{c l}-1\right)}} \propto T_{c}^{-\frac{z_{c l}-1}{z}}
$$

A potential candidate for the 3D-QSN transition is the Ginzburg-Landau theory proposed by Herbut 66. It describes the disordered d-wave superconductor to disordered metal transition at weak coupling and is characterized by the critical exponents $z=2$ and $\bar{\nu}=1 / 2$, except in an exponentially narrow region. Although the overdoped cuprates included in Fig.11 are still quite far from the overdoped limit, the upturn of the data for slightly overdoped $\mathrm{YBa}_{2} \mathrm{Cu}_{3} \mathrm{O}_{7-\delta}(\mathrm{YBCO})$, signals with the scaling relation (11) the flow to 3D-QSN criticality with $z_{c l}>1$, as indicated in Fig.1. Thus unlike the organic superconductors the cuprates undergo a doping tuned crossover from 2D-QSI to 3D-QSN criticality, where $\sigma_{c}^{D C}\left(T_{c}^{+}\right)$and $\lambda_{c}(0)$ tend to infinity, while $T_{c}$ vanishes. These critical points are attained in the underdoped and overdoped limit, respectively, where $T_{c}$ vanishes. In this crossover cuprates pass either to the organics line or the dotted one, spanned by conventional superconductors (see Fig.1. The $\mathrm{YBa}_{2} \mathrm{Cu}_{3} \mathrm{O}_{7-\delta}(\mathrm{YBCO})$ and $\mathrm{La}_{2-x} \mathrm{Sr}_{x} \mathrm{CuO}_{4+\delta}$ (LSCO) cross the dotted mean-field line in the neighborhood of $\mathrm{TaS}_{2}, \mathrm{Sr}_{2} \mathrm{RuO}_{4}$ and $\mathrm{MgB}_{2}$. Since $\mathrm{Sr}_{2} \mathrm{RuO}_{4}$ exhibits Fermi liquid normal state properties [67, fluctuations do not play an essential role in this region of the $\lambda_{c}(0)-\sigma_{c}^{D C}\left(T_{c}^{+}\right)$plane. In the heavily overdoped regime, however, the flow to the 3D-QSN transition (red arrow) enhances quantum fluctuations and invalidates mean-field concepts. On the contrary, the organic superconductors evolve rather smoothly from the mean-field regime, to which $\mathrm{Pb}, \mathrm{Nb}, \mathrm{Nb}$-junctions, $\alpha-\mathrm{Mo}_{1-x} \mathrm{Ge}_{x}$ and the dichalcogenides belong, to 2D-QSI criticality. Thus, the flow to 2D-QSI and 3D-QSN criticality and the associated crossovers give a clear perspective of the regimes where quantum fluctuations are essential. Since the order parameter is a complex scalar, the finite temperature critical behavior falls into the 3D-XY universality class [1,7]. Due to the existence of the 2D-QSI and 3D-QSN critical points, and of the mean-field line, its detection will be hampered by the associated crossovers which reduce the temperature regime where thermal 3D-XY fluctuations dominate. Nevertheless, our analysis clearly revealed that superconductivity in the organic and cuprate superconductors is a genuine 3D phenomenon and that the competition between anisotropy and superconductivity destroys the latter in 2D limit, even in the ground state. When the aforementioned universality classes of the 2D-QSI and 3D-QSN transitions hold true, 
disorder plays an essential role. At 2D-QSI criticality it localizes the pairs and destroys superfluidity 54,55] and at the 3D-QSN transition it destroys, combined with a weakening of the pairing interaction, superfluidity and pairs [66]. A detailed account of the flow from mean-field to 2D-QSI criticality in organics and the crossover from the 2D-QSI to the 3D-QSN critical point is a challenge for microscopic theories attempting to solve the puzzle of superconductivity in these materials.

\section{ACKNOWLEDGMENTS}

The author is grateful to A.R. Bishop, H.Keller, K.A. Müller and J. Roos for suggestions on the subject matter.

[1] T. Schneider and J. M. Singer, Phase Transition Approach To High Temperature Superconductivity, Imperial College Press, London, 2000, and references therein.

[2] M. Lang, F. Steglich, N. Toyata and T. Sasaki, Phys. Rev. B 49, 15227 (1994), and references therein.

[3] T. Shibauchi, M. Sato, S. Ooi and T. Tamegai, Phys.Rev. B 57, 5622 (1998)

[4] N. J. Clayton et al., cond-mat/0108376

[5] S.V. Dordevic et al., cond-mat/0102455

[6] S.V. Dordevic, E.J. Singley, D.N. Basov, S. Komiya and Ando Y., cond-mat/0112042

[7] T. Schneider, cond-mat/0204236

[8] T. Motohashi et al., Phys. Rev. B 61, 9269 (2000)

[9] D.N. Basov, T. Timusk, B. Dabrowski and J.D. Jorgensen, Phys. Rev. B 50, 3511 (1994)

[10] C.C. Homes, T. Timusk, D.A. Bonn, R. Liang and W. Hardy, Physica C 254, 1(1995)

[11] S. Uchida and K. Tamasaku, Physica C 293, 1 (1997)

[12] J. Schutzmann, S. Tajima, S. Miyamoto and S. Tanaka, Phys.Rev.Lett. 73, 174 (1994)

[13] C. Bernhard et al. Phys. Rev. B 59, 6631 (1999)

[14] D.N. Basov, H.A. Mook, B. Dabrowski and T. Timusk, Phys. Rev. B 52, 13141 (1995)

[15] S. Uchida, K. Tamasaku and S. Tajima, Phys.Rev. B 53, 14558 (1996)

[16] J.R. Kirtley, K.A. Moler, G. Villard and A. Maignan, Phys.Rev.Lett. 81, 2140 (1998)

[17] D. N. Basov et al., Science 288, 468 (2000)

[18] K.A. Moler, J. R. Kirtley, D.G. Hinks, T.W. Li and M. Xu, Science 279, 1193 (1998)

[19] A. Katz et al., Phys. Rev. B 61, 5930 (2000)

[20] J. R. Cooper, L. Forro and B. Keszei, Nature 343, 444 (1990)

[21] A. Pimenov et al., Phys.Rev. B 62, 9822 (2000)

[22] P. Garoche, J.J. Veyssie, P. Manuel and P. Molinie, Sol. Stat. Comm. 19 , 455 (1976)

[23] J.J. Finley and B.S. Deaver, Sol. Stat. Comm. 36, 493 (1980)

[24] P. de Trey, S. Gygaz and J.-P. Jan, J.Low Temp. Phys, 11, 421 (1973)

[25] K. Onabe, M. Naito and S. Tanaka, J. Phys .Soc.Jap. 45, 50 (1978)

[26] R.J.Kennedy et al., Can. J. Phys. 62, 776 (1984)

[27] A.H. Thompson, F. R. Gamble and R.F. Koehler, Phys.Rev. B 5, 2811 (1972)

[28] X. Su, F. Zuo, J.A. Schlueter, A. Kini. and J.M. Williams, Phys.Rev. B 58 , 2944 (1998)

[29] A. Carrington et al., Phys.Rev.Let. 83, 4172 (1999)

[30] T. Shibauchi, M. Sato, A. Mashio, T. Tamegai, H. Mori, S. Tajima and S. Tanaka, Phys. Rev. B 55, 11979 (1997)

[31] M. Dressel, O. Klein, G. Gruner, K.D. Carlson, H. Wang and J.M. Williams, Phys.Rev. B 50, 13603 (1994)

[32] H. Taniguchi, H. Sato, Y. Nakazawa and K. Kanoda, Phys.Rev. B 53, 8879 (1996)

[33] S. Wanka, D. Beckmann, J. Wosnitza, E. Balthes, D. Schweitzer, W. Strunz and H.J. Keller, Phys.Rev. B 53, 9301 (1996)

[34] K. Kajita, Y. Nishio, S. Moriyama, W. Sasaki, R.Kato, H. Kobayashi and A. Kobayashi, Sol.Stat.Comm. 64, 1279 (1987)

[35] X. Su, F. Zuo, J.A. Schlueter, A.M. Kini and J.A. Williams, Phys.Rev. B 59 , 4376 (1999)

[36] R. Prozorov et al. Phys. Rev. B 63, 052506 (2001)

[37] Y. Maeno, H. Hahimoto, Y. Yoshida, S. Nishizaki, T. Fujita, J.G. Bednorz and F. Lichtenberg, Nature 372,532 (1994)

[38] K. Yoshida, Y. Maeno, S. Nishizaki and T. Fujita, Physica C 263, 519 (1996)

[39] C. Panagopoulos et al., cond-mat/0103060

[40] M. Angst et al., cond-mat/0112166

[41] D.K. Finnemore et al. Phys.Rev.Lett. 86, 2420 (2001) 
[42] A.V. Pronin, M. Dressel, A. Pimenov, A. Loidl, L.V. Rosheim and L.H. Greene, Phys.Rev.B 57, 14416 (1998)

[43] O. Klein, E.J. Nicol, K. Holzer and G. Gruner, Phys.Rev. B 50, 6307 (1994)

[44] E.Goldobin et al. , Phys.Rev. B 58, 15078 (1998)

[45] S.J. Turneaure, T.R. Lemberger and J.M. Graybeal, Phys.Rev.Lett. 84, 987 (2000)

[46] M. Tinkham, Introduction to Superconductivity (Mc Graw Hill, New York, 1975)

[47] L. N. Bulaevskii, Sov. Phys. JETP 37, 1133 (1973)

[48] V. Ambegaokar and A. Baratoff, Phys. Rev. Lett. 10, 486 (1963)

[49] G. Deutscher and O. Entin-Wohlman, J. Phys. C 10, L433 (1977)

[50] L. Onsager, Phys. Rev. 65, 117 (1944)

[51] K.Kim and P.B.Weichman, Phys.Rev. B 43, 13583 (1991)

[52] T. Schneider and H. Keller, Phys.Rev.Lett. 86, 4899 (2001)

[53] T.Schneider and J.M.Singer, J.of Superconductivity 13, 789 (2000)

[54] M.P.A. Fisher, P.B. Weichman, G. Grinstein and D.S. Fisher, Phys.Rev.Lett. 64, 587 (1990)

[55] I.F. Herbut, Phys.Rev. B61, 14723 (2000)

[56] Y. Nakazawa, Y. Nakazawa, H. Taniguchi, A. Kawamoto and K. Kanoda, Phys.Rev. B 61, 16295 (2000)

[57] S. Kawamata et al., J. Low. Temp. Phys. 105, 1721 (1996)

[58] H. Taniguchi, H. Sato, Y. Nakazawa and H. Kanoda, Phys.Rev. B 57, 3623 (1998)

[59] Y.J. Uemura et al., Phys. Rev. B 38, 909 (1988), Phys. Rev. Lett. 62, 2317 (1989)

[60] M.I. Larkin et al., Phys. Rev. B 64, 144514(2001)

[61] P. A. Crowell, F. W van Keuls and J. R. Reppy, Phys.Rev. B 55, 12620 (1997)

[62] Y. Fukuzumi, K. Mizuhashi, K. Takenaka and S. Uchida, Phys.Rev.Lett. 76, 684 (1996)

[63] K. Semba and A. Matsuda, Phys.Rev.Lett. 86, 496 (2000)

[64] C. Bernhard et al., Phys. Rev. Lett. 86, 1614 (2001)

[65] C. Niedermayer et al., Phys. Rev. Lett. 71, 1764 (1993)

[66] I. F. Herbut, Phys. Rev. Lett. 85, 1532 (2000)

[67] C. Bergemann, S.R. Julian, A.P. Mackenzie, S. Nishizaki and Y. Maeno, Phys.Rev.Lett. 84, 2662 (2000), and references therein. 